\title{
Open-charm mesons in nuclear matter at finite temperature beyond the zero-range approximation
}

\author{
C. E. Jiménez-Tejero, ${ }^{1}$ A. Ramos, ${ }^{1}$ L. Tolós, ${ }^{2}$ and I. Vidaña ${ }^{3}$ \\ ${ }^{1}$ Departament d'Estructura i Constituents de la Matèria and Institut de Ciències del Cosmos, Universitat de Barcelona, Avda. Diagonal 647, \\ E-08028 Barcelona, Spain \\ ${ }^{2}$ Institut de Ciències de l'Espai (IEEC/CSIC), Campus Universitat Autònoma de Barcelona, Facultat de Ciències, Torre C5, \\ E-08193 Bellaterra, Spain \\ ${ }^{3}$ Centro de Física Computacional, Department of Physics, University of Coimbra, PT-3004-516, Coimbra (Portugal)
}

(Received 23 February 2011; revised manuscript received 30 May 2011; published 18 July 2011)

\begin{abstract}
The properties of open charm mesons, $D, \bar{D}, D_{s}$, and $\bar{D}_{s}$ in nuclear matter at finite temperature are studied within a self-consistent coupled-channel approach. The interaction of the low-lying pseudoscalar mesons with the ground-state baryons in the charm sector is derived from a $t$-channel vector-exchange model. The in-medium scattering amplitudes are obtained by solving the Lippmann-Schwinger equation at finite temperature including Pauli blocking effects, baryon dressing, as well as $D, \bar{D}, D_{s}$, and $\bar{D}_{s}$ self-energies taking their mutual influence into account. We find that the in-medium properties of the $D$ meson are affected by the $D_{s}$-meson self-energy through the intermediate $D_{s} Y$ loops coupled to $D N$ states. Similarly, dressing the $\bar{D}$ meson in the $\bar{D} Y$ loops has an influence over the properties of the $\bar{D}_{s}$ meson.
\end{abstract}

DOI: 10.1103/PhysRevC.84.015208

PACS number(s): 14.40.Lb, 14.20.Lq, 21.65.-f, 12.38.Lg

\section{INTRODUCTION}

Over the past years the properties of charmed hadrons have received a lot of attention in connection with experiments in lepton colliders (CLEO, Belle, BaBar) and hadron facilities (CDF at Fermilab, PHENIX and STAR at RHIC, and the forthcoming PANDA and CBM experiments at FAIR) [1-22]. The discovery of new resonances with charm content has sparked the interest of not only many experimental but also theoretical research groups in order to find plausible explanations for the nature of the newly found states.

The study and characterization of resonances has been a very active topic of research in hadron physics during the last decades. The goal is to establish whether some resonances have the genuine $q \bar{q}$ or $q q q$ structure predicted by the quark model $[23,24]$ or, alternatively, qualify better as hadron molecules generated dynamically. A series of pioneer works [25-30], based on a $t$-channel vector-meson exchange (TVME) force and more recent approaches in terms of chiral Lagrangians [31-49] have proven to be very promising and successful in describing a wealth of $S$-wave baryonic resonances in the light $\mathrm{SU}(3)$ sector within the molecular picture using coupled-channel dynamics with effective hadronic degrees of freedom. In the modern language of chiral Lagrangians those states emerge from the scattering of the $0^{-}$octet Goldstone bosons off baryons of the $1 / 2^{+}$octet. Moreover, $P$ and $D$-wave baryonic resonances have been generated based on the SU(3) leading-order chiral Lagrangian by means of meson scattering off baryons of the $3 / 2^{+}$decuplet [50-53] and incorporating vector-meson degrees of freedom [54-59]. Molecular states of two pseudoscalar mesons and one baryon [60-64] have also been studied to interpret low-lying $1 / 2^{+}$ states. All these results sustain the so-called hadrogenesis conjecture, formulated by Lutz and Kolomeitsev a few years ago, according to which resonances not belonging to the large $N_{c}$ ground state of QCD are generated by coupled-channel dynamics $[42,43]$.
Recently, the charm degree of freedom has been incorporated in those unitarized coupled-channel approaches to describe open- and hidden-charm mesons [65-70]. Similar methods have been used in the meson-baryon sector [71-75], partially motivated by the parallelism between the $\Lambda(1405)$ and the $\Lambda_{c}(2595)$. The meson-baryon interaction in the charm sector is constructed using the $t$-channel exchange of vector mesons between pseudoscalar mesons and baryons and performing the zero-range approximation while preserving chiral symmetry for light mesons [73]. The extension to $D$ wave $J=3 / 2^{-}$resonances was first attempted by extending the basis to include the $J=3 / 2^{+}$baryons [76]. In order to be consistent with the spin-flavor heavy-quark symmetry (HQS) [77-79], the vector mesons were incorporated later within a SU(8)-inspired model [80,81], similar to the SU(6) one developed in the light sector $[55,56]$. An alternative approach based on the local hidden gauge formalism has recently become available [82]. On the other hand, there have been also attempts to construct the $D N$ and $\bar{D} N$ interaction by incorporating the charm degree of freedom in the SU(3) meson-exchange model of the Jülich group [83-85].

Nuclear medium modifications have been lately incorporated as a second step. The aim is to further investigate the nature of resonant states, such as the $\Lambda_{c}(2595)$, but also to test the dynamics of charmed hadrons with nucleons and nuclei. The properties of open-charm mesons in nuclear matter can influence the charmonium production yield in hot dense matter, which is one of the observables that might indicate the formation of the quark-gluon plasma phase of QCD at high density and temperature [86]. Another exciting scenario is the possible formation of $D$-mesic nuclei $[87,88]$ and of exotic nuclear bound states like $J / \Psi$ in nuclei [89-91]. From the experimental side, the physics program of the CBM experiment as well as part of the PANDA collaboration at FAIR [92] will be devoted to the properties of open and hidden charm in dense matter. In particular, the physics goal is to extend to the heavy-quark sector the GSI program for in-medium 
modifications of hadron properties in the light sector and to provide insight into the charm-nucleus interaction.

Works based on mean-field approaches provided important shifts for the $D$ and $\bar{D}$ open-charm meson masses [93-96], which alters the formation of charmonium substantially [97]. Some of those models have been recently revised [98-100]. A different perspective is offered by models that, working within coupled-channel unitarized schemes, go beyond mean field and provide the spectral features of the charm mesons in symmetric nuclear matter at zero [74,75,101] and finite temperature [102,103]. Lately, this meson-baryon basis has been extended to incorporate HQS. In this way, not only $D$ meson but also $D^{*}$-meson features have been studied [104].

A common feature of the previous models is the use of an interaction kernel in the zero-range approximation $(t \rightarrow 0)$. This is justified for diagonal amplitudes close to threshold and for nondiagonal transition amplitudes where the masses of mesons and baryons in the initial and final meson-baryon states differ moderately. However, the charm-exchange processes, for which the difference in masses between the external mesons is comparable with the mass of the charmed vector meson being exchanged, point toward the breakdown of the zero-range approximation. Charmed baryon resonances have been studied using the full $t$ dependence of the $t$-channel vector-exchange driving term in Ref. [105]. Compared to the previous TVME local models, where the $t \rightarrow 0$ limit was implemented, the work of Ref. [105] obtained the same amount of resonances but located in general at somewhat higher energies and having larger widths. Some of these resonances could clearly be identifiable with experimentally seen states, such as $\Lambda_{c}(2595), \Sigma_{c}(2800), \Xi_{c}(2790)$, and $\Xi_{c}(2980)$.

In the present work, we study the behavior of the dynamically generated baryonic resonances in hot dense matter, as well as the spectral features of the open charm mesons $\left(D, \bar{D}, D_{s}\right.$, and $\left.\bar{D}_{s}\right)$, within a self-consistent coupled-channel approach that considers the full $t$-dependent TVME interaction kernel employed in Ref. [105]. We pay a particular attention to the influence that the dressed mesons exert on each other. We find that the simultaneous dressing of the charm mesons $\left(D, D_{s}\right)$ in the $C=1$ sector, or the anticharm mesons $\left(\bar{D}, \bar{D}_{s}\right)$ in the $C=-1$ one, affects their in-medium properties in a nonnegligible way.

The article is organized as follows. In Sec. II we present the formalism. We first revise the model adopted for the free space amplitudes, and, next, we describe the modifications that incorporate the medium effects. Our results for the medium modified resonances and for the spectral functions of the opencharm mesons at various densities and temperatures are shown in Sec. III. A summary of our conclusions is presented in Sec. IV.

\section{FORMALISM}

In this section we will first review briefly the coupledchannel approach employed in our previous work [105], where we studied open-charm baryon resonances dynamically generated from the free-space interaction of the lowlying pseudoscalar mesons with the ground-state baryons using a $t$-channel vector-exchange driving force. After that, we will introduce the main sources of medium effects, and we will implement them in our coupled-channel formalism.

Since the properties of the $D, \bar{D}, D_{s}$, and $\bar{D}_{s}$ mesons in a hot and dense environment will be determined, respectively, from the $D N, \bar{D} N, D_{s} N$, and $\bar{D}_{s} N$ amplitudes, we list in Table I the corresponding set of coupled channels in each of the related isospin $(I)$, strangeness $(S)$, and charm $(C)$ sectors.

\section{A. Free-space coupled-channel approach}

The free-space amplitudes $T$ that describe the scattering of the pseudoscalar meson fields off the ground-state baryon fields can be obtained by solving the well-known LippmannSchwinger equation, which schematically reads

$$
T=V+V J T \text {. }
$$

The loop function $J$ is the product of the meson and baryon single-particle propagators, and the scattering kernel $V$ describes the interaction between the pseudoscalar mesons and the ground-state baryons. Following the original work of Hofmann and Lutz [73], we identify a $t$-channel exchange of vector mesons as the driving force for the $S$-wave scattering between pseudoscalar mesons in 16-plet and baryons in 20-plet representations. The scattering kernel takes the form (see Ref, [73] for details)

$$
\begin{aligned}
V_{i j}^{(I, S, C)}\left(p_{i}, q_{i}, p_{j}, q_{j}\right)= & \frac{g^{2}}{4} \sum_{V \in[16]} C_{i j ; V}^{(I, S, C)} \bar{u}\left(p_{j}\right) \gamma^{\mu} \\
& \times\left(g_{\mu \nu}-\frac{\left(q_{i}-q_{j}\right)_{\mu}\left(q_{i}-q_{j}\right)_{v}}{m_{V}^{2}}\right) \\
& \times \frac{1}{t-m_{V}^{2}}\left(q_{i}+q_{j}\right)^{v} u\left(p_{i}\right)
\end{aligned}
$$

where the sum runs over all vector mesons of the SU(4) 16-plet, $\left(\rho, K^{*}, \bar{K}^{*}, \omega, \phi, D^{*}, D_{s}^{*}, \bar{D}^{*}, \bar{D}_{s}^{*}, J / \Psi\right), m_{V}$ is the mass of the exchanged vector meson, $g$ is the universal vector meson coupling constant, $p_{i}, q_{i}, p_{j}$, and $q_{j}$ are the four momenta of the incoming and outgoing baryon and meson, and the coefficients $C_{i j ; V}^{(I, S, C)}$ denote the strength of the interaction in the different $(I, S, C)$ sectors and meson-baryon channels $(i, j)$. The value of $g=6.6$ that reproduces the decay width of the $\rho$ meson [106] has been considered in this work. The $S$-wave projection of the scattering kernel is easily obtained, and in the center-of-mass (c.m.) frame it takes the analytical form

$$
\begin{aligned}
V_{i j, l=0}^{(I, S, C)}\left(\vec{k}_{i}, \vec{k}_{j}\right)= & N \frac{g^{2}}{8} \sum_{V \in[16]} C_{i j ; V}^{(I, S, C)} \\
& \times\left[\frac{2 \beta}{b}+\frac{\alpha b-\beta a}{b^{2}} \ln \left(\frac{a+b}{a-b}\right)\right],
\end{aligned}
$$

with $a, b, \alpha$, and $\beta$ being

$$
\begin{aligned}
& a=m_{i}^{2}+m_{j}^{2}-2 \omega_{i}\left(\left|\vec{k}_{i}\right|\right) \omega_{j}\left(\left|\vec{k}_{j}\right|\right)-m_{V}^{2}, \\
& b=2\left|\vec{k}_{i}\right|\left|\vec{k}_{j}\right|
\end{aligned}
$$


TABLE I. Coupled-channel meson-baryon states involved in $D N, \bar{D} N, D_{s} N$, or $\bar{D}_{s} N$ interactions. The energy threshold of each state is given in brackets.

\begin{tabular}{|c|c|c|c|c|c|}
\hline$(I, S, C)$ & & & Channels & & \\
\hline$\left(\frac{1}{2},-1,-1\right)$ & & $\bar{D}_{s} N(2908)$ & $\bar{D} \Lambda(2985)$ & $\bar{D} \Sigma(3062)$ & \\
\hline$(0,0,-1)$ & & & $\bar{D} N(2806)$ & & \\
\hline$(1,0,-1)$ & & & $\bar{D} N(2806)$ & & \\
\hline$(0,0,1)$ & $\begin{array}{l}\pi \Sigma_{c}(2591) \\
D_{s} \Lambda(3085)\end{array}$ & $\begin{array}{c}D N(2806) \\
\eta^{\prime} \Lambda_{c}(3243)\end{array}$ & $\begin{array}{c}\eta \Lambda_{c}(2832) \\
\eta_{c} \Lambda_{c}(5265)\end{array}$ & $\begin{array}{l}K \Xi_{c}(2963) \\
\bar{D} \Xi_{c c}(5307)\end{array}$ & $K \Xi_{c}^{\prime}(3070)$ \\
\hline$(1,0,1)$ & $\begin{array}{l}\pi \Lambda_{c}(2424) \\
K \Xi_{c}^{\prime}(3070)\end{array}$ & $\begin{array}{l}\pi \Sigma_{c}(2591) \\
D_{s} \Sigma(3162)\end{array}$ & $\begin{array}{c}D N(2806) \\
\eta^{\prime} \Sigma_{c}(3410)\end{array}$ & $\begin{array}{l}K \Xi_{c}(2963) \\
\bar{D} \Xi_{c c}(5307)\end{array}$ & $\begin{array}{c}\eta \Sigma_{c}(2999) \\
\eta_{c} \Sigma_{c}(5432)\end{array}$ \\
\hline$\left(\frac{1}{2}, 1,1\right)$ & & $K \Lambda_{c}(2779)$ & $D_{s} N(2908)$ & $K \Sigma_{c}(2946)$ & \\
\hline
\end{tabular}

$$
\begin{aligned}
\alpha= & \Omega_{i}\left(\left|\vec{k}_{i}\right|\right)+\Omega_{j}\left(\left|\vec{k}_{j}\right|\right)-M_{i}-M_{j} \\
& -\frac{m_{j}^{2}-m_{i}^{2}}{m_{V}^{2}}\left(\Omega_{j}\left(\left|\vec{k}_{j}\right|\right)-\Omega_{i}\left(\left|\vec{k}_{i}\right|\right)+M_{i}-M_{j}\right), \\
\beta= & \frac{\left|\vec{k}_{i}\right|\left|\vec{k}_{j}\right|}{\left[E_{i}\left(\left|\vec{k}_{i}\right|\right)+M_{i}\right)\left(E_{j}\left(\left|\vec{k}_{j}\right|\right)+M_{j}\right]} \\
& \times\left[\Omega_{i}\left(\left|\vec{k}_{i}\right|\right)+\Omega_{j}\left(\left|\vec{k}_{j}\right|\right)+M_{i}+M_{j}\right. \\
& \left.-\frac{m_{j}^{2}-m_{i}^{2}}{m_{V}^{2}}\left(\Omega_{j}\left(\left|\vec{k}_{j}\right|\right)-\Omega_{i}\left(\left|\vec{k}_{i}\right|\right)+M_{j}-M_{i}\right)\right],
\end{aligned}
$$

where $\vec{k}_{i}, \vec{k}_{j}$ are the initial and final relative momenta, $m_{i}, m_{j}, M_{i}, M_{j}$ the masses of the incoming and outgoing mesons and baryons, and $\omega_{i}\left(\left|\vec{k}_{i}\right|\right), \omega_{j}\left(\left|\vec{k}_{j}\right|\right), E_{i}\left(\left|\vec{k}_{i}\right|\right), E_{j}\left(\left|\vec{k}_{j}\right|\right)$ their corresponding energies, which have been taken to be their on-shell values. The factor $N=\left[\left(E\left(\left|\vec{k}_{i}\right|\right)+M_{i}\right)\left(E\left(\left|\vec{k}_{j}\right|\right)+\right.\right.$ $\left.\left.M_{j}\right) /\left(4 M_{i} M_{j}\right)\right]^{1 / 2}$ comes from the normalization of the Dirac spinors. We have defined $\Omega(|\vec{k}|) \equiv \omega(|\vec{k}|)+E(|\vec{k}|)$. We note that the zero-range approximation (i.e., $t \rightarrow 0$ ) of the $S$-wave scattering kernel is obtained by expanding the logarithm of Eq. (3) in the limit $b / a \rightarrow 0$ up to the linear term in $b / a$ and setting $a=-m_{V}^{2}$. The interested reader is referred to our previous work [105] for a detailed analysis of the validity of the zero-range approximation.

In Eqs. (2) and (3), we have assumed infinitely (zero-width) exchanged vector mesons, because the value of $t$ is never larger than the square of the minimum energy required for the meson to decay. In other words, since the mesons being exchanged in this problem are largely off shell, they will be treated as stable particles.

Once the scattering kernel has been constructed, one can finally write the $S$-wave projection of the LippmannSchwinger equation,

$$
\begin{aligned}
T_{i j, l=0}^{(I, S, C)}\left(\vec{k}_{i}, \vec{k}_{j}, \sqrt{s}\right)= & V_{i j, l=0}^{(I, S, C)}\left(\vec{k}_{i}, \vec{k}_{j}\right) \\
& +\sum_{k} \int \frac{d \vec{k}}{(2 \pi)^{3}} F(|\vec{k}|) V_{i k, l=0}^{(I, S, C)}\left(\vec{k}_{i}, \vec{k}\right) \\
& \times J_{k}(\sqrt{s}, \vec{k}) T_{k j, l=0}^{(I, S, C)}\left(\vec{k}, \vec{k}_{j}, \sqrt{s}\right),
\end{aligned}
$$

where $\sqrt{s}$ is the total energy in the c.m. frame. The loop function $J$ explicitly reads

$$
\begin{aligned}
J_{k}^{(I, S, C)}(\sqrt{s}, \vec{k})= & \frac{M_{k}}{2 E_{k}(|\vec{k}|) \omega_{k}(|\vec{k}|)} \\
& \times \frac{1}{\sqrt{s}-E_{k}(|\vec{k}|)-\omega_{k}(|\vec{k}|)+i \eta},
\end{aligned}
$$

and $F(|\vec{k}|)$ is a dipole-type form factor,

$$
F(|\vec{k}|)=\left(\frac{\Lambda^{2}}{\Lambda^{2}+|\vec{k}|^{2}}\right)^{2}
$$

which has been introduced to regularize the integral. This form is typically adopted in studies of hadron-hadron interactions within the scheme of Lippmann-Schwinger-type equations in the light flavor sector [107]. The value of the cutoff $\Lambda$ is a free parameter of our model. Given the limited amount of data for charmed baryon resonances, and in order to simplify the analysis, the cutoff $\Lambda$ is adjusted to $903 \mathrm{MeV} / \mathrm{c}$ in order to reproduce the position of the well-known $J^{P}=1 / 2^{-}$ $\Lambda_{c}(2595)$ having $(I, S, C)=(0,0,1)$, and the same value is used for the other sectors explored in this work. In Table II we summarize the position, width, and most important couplings of the dynamically generated states appearing in the various $(I, S, C)$ sectors listed in Table I. Note that there are no resonances in the singled-channel $(I, S, C)=(0,0,-1)$ and $(1,0,-1)$ sectors of the $\bar{D} N$ interaction. In the other $C=-1$ case, having $\left(I=\frac{1}{2}, S=-1\right)$, we find a pole just below the $\bar{D}_{s} N$ threshold. The remaining cases have $C=1$, and, although they were thoroughly analyzed in Ref. [105], we briefly comment here on a few essential characteristics that will be useful for our discussion of the in-medium results in the next section. In the $(I=0, S=0)$ sector, apart from the $\Lambda_{c}(2595)$ resonance to which we fit the model, there is another very narrow one at $2805 \mathrm{MeV}$, just below the threshold for $D N$ states but coupling very little to them. We also predict two narrow resonances in the $(I=1, S=0)$ sector at 2551 and $2804 \mathrm{MeV}$, right below the thresholds of the channels to which they couple more strongly, namely, $\pi \Sigma_{c}$ and $D N$, respectively. In the $\left(I=\frac{1}{2}, S=1\right)$ case, we predict a cusplike structure placed at the threshold of $K \Sigma_{c}$, the channel that shows the largest coupling to this state. 
TABLE II. Dynamically generated baryon resonances with open charm in various $(I, S, C)$ sectors for a cutoff momentum $\Lambda=903 \mathrm{MeV} / \mathrm{c}$. The table shows the position $\left(M_{R}\right)$ and width $(\Gamma)$ of the resonance, together with the most important couplings to the meson-baryon channels, as well as the couplings to the channels in which it can decay (marked with an asterisk).

\begin{tabular}{lccc}
\hline \hline$(I, S, C)$ & $M_{R}[\mathrm{MeV}]$ & $\Gamma[\mathrm{MeV}]$ & $|g|($ channel $)$ \\
\hline$\left(\frac{1}{2},-1,-1\right)$ & $2906(\mathrm{pole})$ & 0 & $1.3\left(\bar{D}_{s} N\right), 1.1(\bar{D} \Lambda), 1.9(\bar{D} \Sigma)$ \\
$(0,0,1)$ & 2595 & 0.5 & $0.31\left(\pi \Sigma_{c}\right)^{*}, 11(D N), 6.0\left(D_{s} \Lambda\right), 2.0\left(\eta_{c} \Lambda_{c}\right)$ \\
$(1,0,1)$ & 2805 & 0.01 & $0.04\left(\pi \Sigma_{c}\right)^{*}, 0.27(D N), 2.2\left(\eta \Lambda_{c}\right), 4.3\left(K \Xi_{c}\right), 0.21\left(D_{s} \Lambda\right)$ \\
$\left(\frac{1}{2}, 1,1\right)$ & 2551 & 0.16 & $0.05\left(\pi \Lambda_{c}\right)^{*}, 3.7\left(\pi \Sigma_{c}\right), 1.1(D N), 2.1\left(K \Xi_{c}^{\prime}\right)$ \\
\hline \hline
\end{tabular}

\section{B. Medium effects}

The are two main sources of medium effects to consider: One is a consequence of the Pauli exclusion principle, which prevents the scattering of two baryons into states that are already occupied. The other is related to the fact that the properties of all mesons and baryons are modified in the medium due to their interactions with the Fermi sea of baryons. Pauli blocking, finite temperature effects, and medium modification of the baryon energy spectra can be incorporated in the coupled-channel equations by simply replacing the free baryon propagator by the in-medium one,

$$
\begin{aligned}
G\left(p_{0}, \vec{p}, \rho, T\right)= & \frac{M}{E(|\vec{p}|, T)}\left[\frac{1-n(\vec{p}, \rho, T)}{p_{0}-E(|\vec{p}|, T)+i \epsilon}\right. \\
& \left.+\frac{n(\vec{p}, \rho, T)}{p_{0}-E(|\vec{p}|, T)-i \epsilon}\right],
\end{aligned}
$$

where $\left(p_{0}, \vec{p}\right)$ is the total four-momentum of the baryon in the nuclear matter rest frame and $n(\vec{p}, \rho, T)$ is the usual Fermi-Dirac distribution function. Similarly to the in-medium $D$ meson study of Ref. [103], the nucleon energy spectrum, $E_{N}(|\vec{p}|, T)$, is taken from a Walecka-type $\sigma-\omega \operatorname{model~[108]:~}$

$$
E_{N}(|\vec{p}|, T)=\sqrt{\vec{p}^{2}+\left[M_{N}-\Sigma^{s}(T)\right]^{2}}+\Sigma^{v} .
$$

The values of the nucleon scalar $\left(\Sigma^{s}\right)$ and vector $\left(\Sigma^{v}\right)$ selfenergies for the densities and temperatures explored in this work are given in Table III. The hyperon $(Y)$ and charmed baryon $\left(Y_{c}\right)$ energy spectra are similarly given as

$$
E_{Y_{(c)}}(|\vec{p}|, T)=\sqrt{\vec{p}^{2}+\left[M_{Y_{(c)}}-\Sigma_{Y_{(c)}}^{s}(T)\right]^{2}}+\Sigma_{Y_{(c)}}^{v} .
$$

Assuming that the $\sigma$ and $\omega$ fields couple only to the $u$ and $d$ quarks, the scalar and vector self-energies of the hyperons and charmed baryons can be written in terms of $\Sigma^{s}$ and $\Sigma^{v}$, as

$$
\Sigma_{Y_{(c)}}^{s}=\frac{2}{3} \Sigma^{s}, \quad \Sigma_{Y_{(c)}}^{v}=\frac{2}{3} \Sigma^{v} .
$$

The resonances that play a relevant role in the properties of the open charm mesons do not couple significatively to baryons having only one light ( $u$ or $d$ ) quark. Therefore, we disregard the dressing of this type of baryons $\left(\Xi_{c}, \Xi_{c}^{\prime}, \Xi_{c c}\right)$.

The nuclear medium effects on the mesons can be incorporated by including their corresponding self-energies,
$\Pi_{m}\left(q_{0}, \vec{q}, \rho, T\right)$, in the meson propagator

$$
D_{m}\left(q_{0}, \vec{q}, \rho, T\right)=\frac{1}{q_{0}^{2}-\vec{q}^{2}-m_{m}^{2}-\Pi_{m}\left(q_{0}, \vec{q}, \rho, T\right)},
$$

$\left(q_{0}, \vec{q}\right)$ being the four-momentum of the meson. This is done in practice through the corresponding Lehmann representation of the meson propagator

$$
\begin{aligned}
D_{m}\left(q_{0}, \vec{q}, \rho, T\right)= & \int_{0}^{\infty} \frac{S_{m}(\omega, \vec{q}, \rho, T)}{q_{0}-\omega+i \epsilon} d \omega \\
& -\int_{0}^{\infty} \frac{S_{\bar{m}}(\omega, \vec{q}, \rho, T)}{q_{0}+\omega-i \epsilon} d \omega,
\end{aligned}
$$

where $S_{m(\bar{m})}(\omega, \vec{q}, \rho, T)$ is the spectral function of the meson $m($ antimeson $\bar{m})$ :

$$
\begin{aligned}
S_{m}\left(q_{0}, \vec{q}, \rho, T\right) & =-\frac{1}{\pi} \operatorname{Im}\left[D_{m}\left(q_{0}, \vec{q}, \rho, T\right)\right] \\
& =-\frac{1}{\pi} \frac{\operatorname{Im}\left[\Pi_{m}\left(q_{0}, \vec{q}, \rho, T\right)\right]}{\left|q_{0}^{2}-\vec{q}^{2}-m_{m}^{2}-\Pi_{m}\left(q_{0}, \vec{q}, \rho, T\right)\right|^{2}} .
\end{aligned}
$$

We note here that in this work only the $D, \bar{D}, D_{s}$, and $\bar{D}_{s}$ mesons have been dressed by self-energy insertions. Mesons $\pi, K, \eta, \eta^{\prime}$, and $\eta_{c}$ have not been dressed, as done, e.g., in Refs. $[75,103,104]$. The reason is that the states containing these mesons couple weakly to the $D N$ and $D_{s} N$ ones and, therefore, it is expected that approximating the $\pi, K, \eta, \eta^{\prime}$ spectral functions by the free-space ones, i.e., delta functions, will not influence much the in-medium properties of the $D$ and $D_{s}$ mesons. We emphasize, however, that the present work addresses for the first time the simultaneous dressing of the $D$ and $D_{s}$ mesons in the charm $C=1$ sector, and that of the $\bar{D}$ and $\bar{D}_{s}$ mesons in the charm $C=-1$ one.

TABLE III. Nucleon scalar $\left(\Sigma^{s}\right)$ and vector $\left(\Sigma^{v}\right)$ self-energies for the densities and temperatures explored in this work. The last columns show the value of the baryon self-energy at zero momentum.

\begin{tabular}{lccccc}
\hline \hline$\rho\left(\mathrm{fm}^{-3}\right)$ & $T(\mathrm{MeV})$ & $\Sigma^{s}(\mathrm{MeV})$ & $\Sigma^{v}(\mathrm{MeV})$ & $\Sigma^{v}-\Sigma^{s}$ & $\Sigma_{Y_{(c)}}^{v}-\Sigma_{Y_{(c)}}^{s}$ \\
\hline 0.16 & 0 & 360.1 & 281.7 & -78.4 & -52.3 \\
0.16 & 100 & 304.7 & 281.7 & -23 & -15.3 \\
0.32 & 0 & 496.5 & 421.5 & -75 & -50 \\
0.32 & 100 & 428.8 & 421.5 & -7.3 & -4.9 \\
\hline \hline
\end{tabular}


The loop function for the free case given by Eq. (6) must now be replaced by the one including the medium and temperature effects on the baryon and meson propagators, as given by Eqs. (8) and (13). Using the Imaginary Time (or Matsubara) Formalism [109], we obtain

$$
\begin{aligned}
J_{k}^{(I, S, C)}\left(P_{0}, \vec{P}, \vec{k}, \rho, T\right) \\
=\frac{M_{k}}{E_{k}(|x \vec{P}+\vec{k}|, T)}\left(\int_{0}^{\infty} d \omega S_{m}(\omega, y \vec{P}-\vec{k}, \rho, T)\right. \\
\quad \times \frac{1-n(x \vec{P}+\vec{k}, \rho, T)+f(\omega, T)}{P_{0}-\omega-E_{k}(|x \vec{P}+\vec{k}|, T)+i \epsilon} \\
\quad+\int_{0}^{\infty} d \omega S_{\bar{m}}(\omega, y \vec{P}-\vec{k}, \rho, T) \\
\left.\quad \times \frac{n(x \vec{P}+\vec{k}, \rho, T)+f(\omega, T)}{P_{0}+\omega-E_{k}(|x \vec{P}+\vec{k}|, T)-i \epsilon}\right),
\end{aligned}
$$

where $P_{0}=q_{0}+E_{k}(|\vec{p}|), \vec{P}=\vec{q}+\vec{p}$, and $\vec{k}=y \vec{p}-x \vec{q}$, with $x=M_{k} /\left(m_{k}+M_{k}\right)$ and $y=m_{k} /\left(m_{k}+M_{k}\right)$, are the total energy, total momentum, and relative momentum of the meson-baryon pair in the nuclear matter rest frame, $n$ is the Fermi distribution of the baryon, and $f$ is the Bose enhancement factor of the meson. In practice, given the nuclear densities and temperatures explored in the present work, we can set $f=0$ for all mesons and $n=0$ for all baryons except for nucleons. One might argue that the Bose enhancement factor for the pions should not be ignored. However, as tested in Ref. [103], the $D N$ amplitudes are insensitive to this factor due to the reduced coupling to $\pi \Sigma_{c}$ states resulting from the heavy mass of the meson exchanged in the transition potential.

The in-medium scattering amplitudes $T$ are obtained by directly solving the coupled-channel Eq. (5) with the medium modified loop function $J_{m}^{(I, S, C)}\left(P_{0}, \vec{P}, \vec{k}, \rho, T\right)$. The in-medium self-energies for the $D, \bar{D}, D_{s}$, and $\bar{D}_{s}$ mesons are then obtained by integrating the in-medium diagonal scattering amplitudes over the nucleon Fermi sea as

$$
\begin{aligned}
\Pi_{D(\bar{D})}\left(q_{0}, \vec{q}, \rho, T\right)= & \int \frac{d^{3} p}{(2 \pi)^{3}} n(\vec{p}, \rho, T) \\
& \times\left[T_{D(\bar{D}) N}^{(I=0)}\left(P_{0}, \vec{P}, \rho, T\right)\right. \\
& \left.+3 T_{D(\bar{D}) N}^{(I=1)}\left(P_{0}, \vec{P}, \rho, T\right)\right],
\end{aligned}
$$

for $D$ and $\bar{D}$, and as

$$
\begin{aligned}
\Pi_{D_{s}\left(\bar{D}_{s}\right)}\left(q_{0}, \vec{q}, \rho, T\right)= & 4 \int \frac{d^{3} p}{(2 \pi)^{3}} n(\vec{p}, \rho, T) \\
& \times T_{D_{s}\left(\bar{D}_{s}\right) N}^{(I=1 / 2)}\left(P_{0}, \vec{P}, \rho, T\right),
\end{aligned}
$$

for $D_{s}$ and $\bar{D}_{s}$.

Finally, we note that the self-energies $\Pi_{m} \quad(m=$ $\left.D, \bar{D}, D_{s}, \bar{D}_{s}\right)$ must be determined in a self-consistent way since they are obtained from the in-medium scattering amplitudes $T_{D N}, T_{\bar{D} N}, T_{D_{s} N}$, and $T_{\bar{D}_{s} N}$, which contain the loop functions $J_{D N}^{(I, S, C)}, J_{D_{s} Y}^{(I, S, C)}(D N$ case $), J_{\bar{D} N}^{(I, S, C)}(\bar{D} N$ case $)$, $J_{D_{s} N}^{(I, S, C)}\left(D_{s} N\right.$ case $)$, and $J_{\bar{D}_{s} N}^{(I, S, C)}, J_{\bar{D} Y}^{(I, S, C)}\left(\bar{D}_{s} N\right.$ case $)$, that are themselves functions of the self-energies $\Pi_{m}$.

\section{RESULTS AND DISCUSSION}

We will start discussing our results for the $C=1$ mesons, $D$ and $D_{s}$. First, we note that their in-medium properties will be influenced by the charm $C=1$ baryonic resonances that couple significatively to $D N$ and $D_{s} N$. From the results of our previous work [105], summarized in Table II, we find the well-known $\Lambda_{c}(2595)$, coupling very strongly to $D N$ states in the $(I=0, S=0)$ sector, and two other resonances, $\Sigma_{c}(2551)$ and $\Sigma_{c}(2804)$, coupling also significatively to $D N$ in the sector $(I=1, S=0)$. The cusplike structure found in the $(I=1 / 2, S=1)$ sector shows also a sizable coupling to $D_{s} N$ states.

In Fig. 1 we show the imaginary part of the $D N$ amplitude at normal nuclear matter saturation density, $\rho_{0}=0.17 \mathrm{fm}^{-1}$,

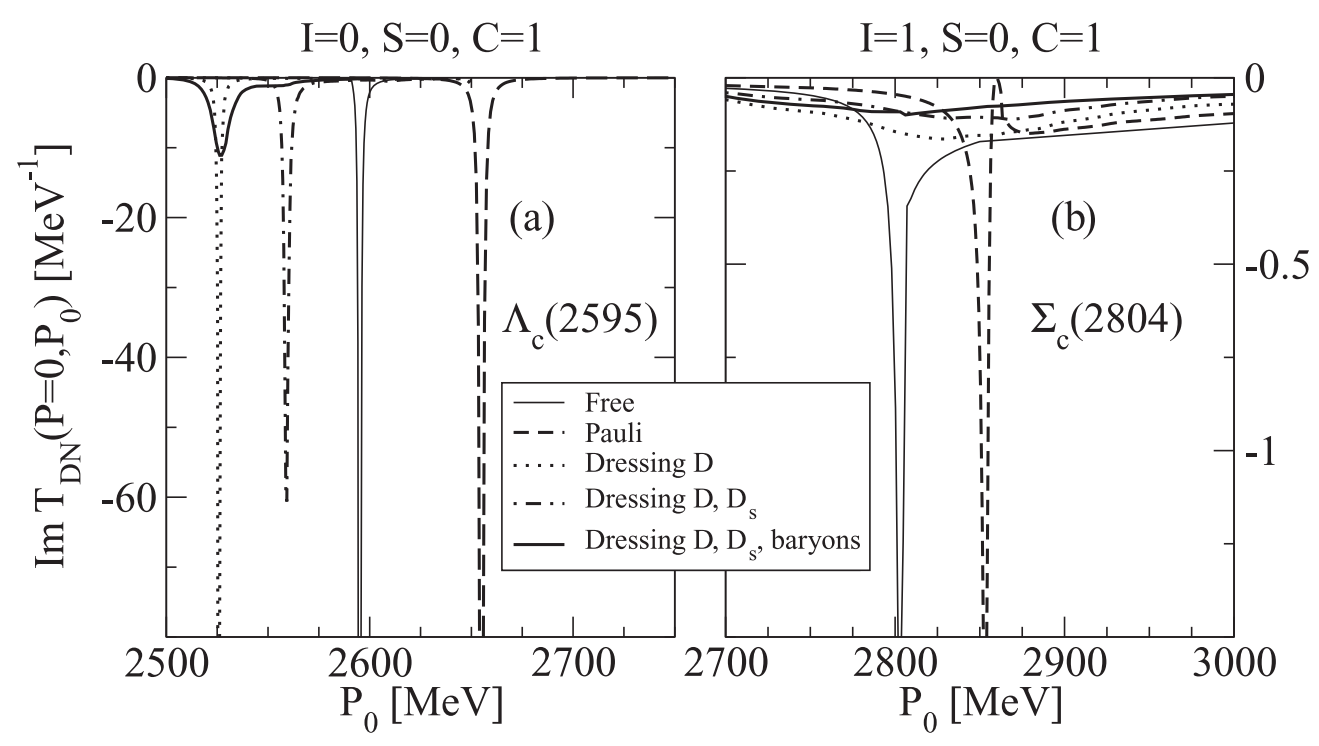

FIG. 1. Imaginary part of the $I=0$ (left panel) and $I=1$ (right panel) $D N \rightarrow D N$ scattering amplitudes in nuclear matter at normal saturation density $\rho_{0}$ and zero temperature, as a function of the total energy $P_{0}$, for a total momentum $\vec{P}=0$ and various approximations. 
and zero temperature, as a function of the center-of-mass energy $P_{0}$, covering an energy range that includes the most relevant resonance in each isospin sector, $I=0$ (left panel) and $I=1$ (right panel), for various approximations. The amplitude in free space $(\rho=0)$ is also shown (thin-solid lines), as a reference. When only Pauli blocking effects are included (dashed line), we observe that the $\Lambda_{c}(2595)$ and $\Sigma_{c}(2804)$ states appear displaced to higher energies, by about 60 and $50 \mathrm{MeV}$, respectively. This repulsive effect is well known and has to do with the loss of phase space associated to the fact that the nucleons are forced to occupy empty states that are located at momentum states above the Fermi momentum. However, when the dressing of the $D$ meson is incorporated self-consistently (dotted line), the $\Lambda_{c}(2595)$ resonance moves to substantially lower energies and the $\Sigma_{c}(2804)$ dilutes. This is naturally explained in terms of the $D$-meson strength distribution, which, as we will see, shows a quasiparticle peak at a lower energy than in free space and a pronounced peak at even lower energies related to $\Lambda_{c}(2595) N^{-1}$ excitations. The reduced in-medium $D N$ threshold opens decay channels for the $\Sigma_{c}(2804)$, which, therefore, broadens considerably. As for the $\Lambda_{c}(2595)$, its position makes it very sensitive to the low-energy strength of the $D$ spectral function. This, together with the large coupling to $D N$ states, explains why the resonance acquires such a large amount of attraction.

In Ref. [75], where the TVME in the $t \rightarrow 0$ limit is employed, a similar behavior is observed for the $\Lambda_{c}(2595)$. The repulsive shift with respect to the free space position due to Pauli blocking effects is compensated by the attractive self-consistent dressing of the $D$ meson. However, the shift is smaller in Ref. [75], as can be seen from Fig. 5 of this reference (model B). Moreover, this TVME $(t \rightarrow 0)$ model also generates a $\Sigma_{c}$ resonance, which lies around $2795 \mathrm{MeV}$ for model B. This structure melts down as the dressing of $D$ mesons is incorporated, because of the opening of new absorption channels, and stays close to the position with only Pauli blocking effects, in agreement with our present results. In the TVME model of Ref. [101] both resonances are generated, but the $\Sigma_{c}$ one is localized at a much smaller energy, around $2620 \mathrm{MeV}$. In that work the self-consistent dressing of $D$ mesons results in an attractive shift for both $\Lambda_{c}$ and $\Sigma_{c}$ dynamically generated resonances, in contrast to our results. Within the SU(8) Weinberg-Tomozawa model of Ref. [80], where heavy-quark spin symmetry is implemented, the $\Sigma_{c}$ state lies around $2900 \mathrm{MeV}$ and has a different spin, $J=3 / 2$. In this scheme, the $\Sigma_{c}$ resonance couples strongly to the $D^{*} N$ channel instead of $D N$ and behaves similarly in matter as the $\Lambda_{c}$ (2595) [104].

It is clear from Table II that the strong coupling of the $\Lambda_{c}(2595)$ resonance to the $D_{s} \Lambda$ channel and that of the $\Sigma_{c}(2804)$ to $D_{s} \Sigma$ states make it advisable to consider also the medium modifications of the $D_{s}$ meson in the intermediate $D_{s} Y$ loops of the $D N$ amplitude. This is a quite arduous task that, to our knowledge, has been attempted for the first time in the present work. Our results, represented by the dash-dotted lines in Fig. 1, clearly show the importance of such dressing. The strength of the dressed $D_{s} Y$ loop gets diluted, making the $\Lambda_{c}(2595)$ to appear $35 \mathrm{MeV}$ higher in energy with respect to the case of considering free $D_{s} Y$ loops.
The changes on the $\Sigma_{c}(2804)$ resonance are more moderate. Finally, including the baryon self-energies (solid line), which produce attraction in the baryon spectrum at $\rho_{0}$ and $T=0$, moves the resonances to lower energies due to the fact that the threshold for intermediate $D N$ states has also been lowered.

The real and imaginary parts of the $D$ and $D_{s}$ selfenergies and spectral functions at normal nuclear matter saturation density and zero temperature are shown in Fig. 2, as functions of the meson energy $q_{0}$, and for a meson momentum $q=0 \mathrm{MeV} / \mathrm{c}$. The approximations displayed include Pauli blocking effects, the additional self-consistent dressing of the given meson, and, in the case of the $D$ meson, the additional dressing of the $D_{s}$ meson in the $D_{s} Y$ intermediate states coupling to $D N$. Results obtained when baryon self-energies are taken into account are also shown.

The features discussed for the $D N$ amplitude in Fig. 1 are also reflected in the imaginary part of the $D$-meson self-energy displayed in the upper left panel of Fig. 2. The middle panel shows the corresponding real part of the self-energy, $\operatorname{Re} \Pi\left(q_{0}, \vec{q}=0\right)$, together with the function $q_{0}^{2}-m_{D}^{2}$ (thin solid line), such that the crossing points of these two functions signal the appearance of pronounced maxima in the spectral function, as long as the imaginary part does not show a pronounced minimum there. Actually, the distribution of the $D$-meson strength shown in the lower-left panel is very rich. All the approximations give a quasiparticle peak located around $35 \mathrm{MeV}$ below the free $D$-meson mass. In addition, each resonance leaving a signature peak in the imaginary part of the self-energy produces a resonant-hole excitation peak in the spectral function, located at a somewhat different value of energy due to the complex structure of the self-energy. The common behavior is that the resonance-hole modes get displaced in the spectral function, moving away from the quasiparticle peak. In the case of Pauli blocking (dashed line) we can clearly distinguish three of such modes in the spectral function, associated with $\Sigma_{c}(2551) N^{-1}, \Lambda_{c}(2595) N^{-1}$, and $\Sigma_{c}(2804) N^{-1}$ excitations. When meson dressing is incorporated, only the $\Lambda_{c}(2595) N^{-1}$ excitation mode is clearly visible. The $\Sigma_{c}(2804) N^{-1}$ mode merges with the quasiparticle peak, and the $\Sigma_{c}(2551) N^{-1}$ one is no longer visible in the spectral function as compared to the $\Lambda_{c}(2595) N^{-1}$ mode. A similar behavior has been observed in Refs. [75,103]. In contrast, in Ref. [101], the $\Sigma_{c}(2804) N^{-1}$ mode appears at a much lower energy and mixes with the $\Lambda_{c}(2595) N^{-1}$ one, while the quasiparticle peak of the $D$ meson experiences a repulsive shift of $32 \mathrm{MeV}$. It is also worth mentioning that in the SU(8)-inspired model of Ref. [104] the quasiparticle peak appears at slightly lower energies than the free mass, but the $D$-meson spectral function shows a completely different shape due to the different resonant-hole composition of the $D$-meson self-energy.

The imaginary part of the $D_{s}$ self-energy, displayed in the upper right panel of Fig. 2, shows only a small enhancement at around $2 \mathrm{GeV}$. This is a reflection of the enhanced cusp found in the $(I=1 / 2, S=1, C=1)$ amplitude at the $K \Sigma_{c}$ threshold [105]. This structure generates a small but nonnegligible amount of strength in the $D_{s}$ spectral function to the right of the quasiparticle peak, which barely moves from its free location. This is in contrast to Ref. [101], where a resonance is 


\section{D meson}

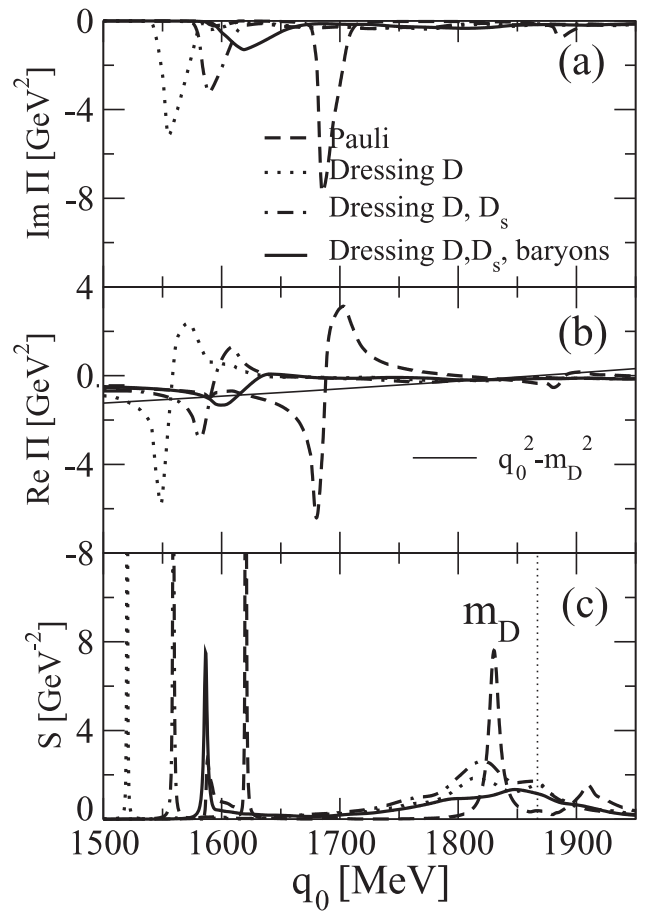

$\mathrm{D}_{\mathrm{s}}$ meson

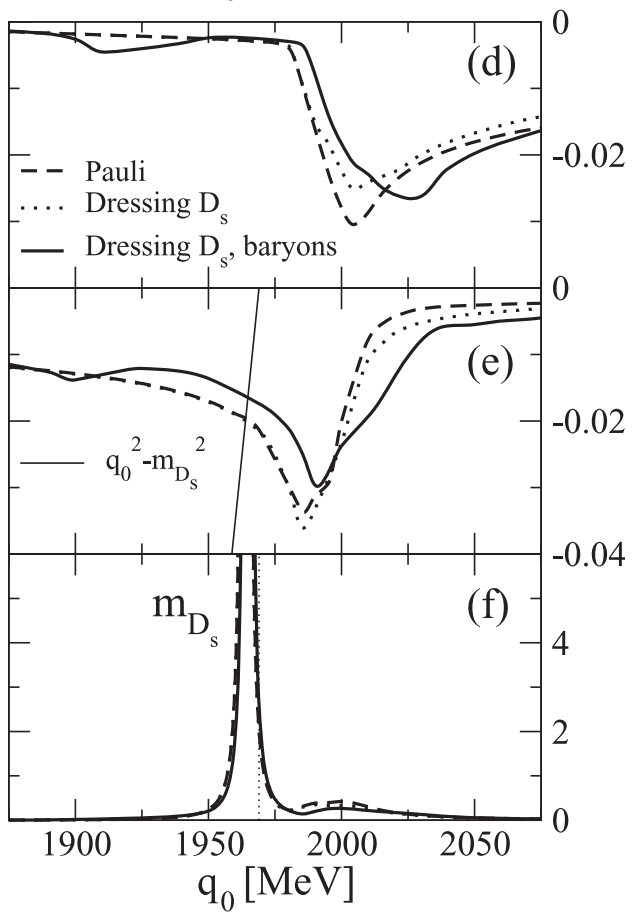

FIG. 2. Imaginary (upper panels) and real (middle panels) parts of the $D$ (left panel) and $D_{s}$ (right panel) meson self-energies and spectral functions (lower panels), as functions of the meson energy $q_{0}$, at normal nuclear matter saturation density, for $q=0 \mathrm{MeV} / \mathrm{c}$ and different approximations. The mass of the $D$ meson and the function $q_{0}^{2}-m_{D}^{2}$ (left panel) and the mass of the $D_{s}$ meson and the function $q_{0}^{2}-m_{D_{s}}^{2}$ (right panel) are shown for comparison.

generated dynamically $75 \mathrm{MeV}$ below the $D_{s} N$ threshold, and the corresponding resonance-hole state in the spectral function appears on the left-hand side of the quasiparticle peak.

In spite of the featureless aspect of the $D_{s}$ spectral function in our model, this relocation of strength from the quasiparticle peak to higher energies diminishes the size of the $D_{s} Y$ loops involved in the coupled-channel problem. Therefore, the simultaneous dressing of the $D$ and $D_{s}$ mesons in our self-consistent coupled-channel model produces a less bound $\Lambda_{c}(2595)$ resonance in nuclear matter, as already shown in Fig. 1. From Fig. 2 we can see that the corresponding $\Lambda_{c}(2595) N^{-1}$ excitation mode of the $D$-meson spectral function appears approximately $40 \mathrm{MeV}$ higher in energy than when only the $D$-meson dressing is considered. Including the dressing of the baryons moves the $\Lambda_{c}(2595) N^{-1}$ structure to higher energies by about $30 \mathrm{MeV}$ because the attraction felt by the $\Lambda_{c}(2595)$ resonance in the medium is smaller than that felt by the dressed nucleon.

The in-medium properties of the $C=-1$ mesons, $\bar{D}$ and $\bar{D}_{s}$, will be determined by the behavior of the corresponding $\bar{D} N$ and $\bar{D}_{s} N$ amplitudes in the nuclear medium. In Fig. 3 we display the imaginary part of the $\bar{D}_{s} N$ amplitude at normal nuclear matter saturation density and zero temperature as a function of the center-of-mass energy $P_{0}$, for various approximations: free (thin-solid line), Pauli blocking (dashed line), the self-consistent calculation including only the dressing of $D_{s}$ (dotted line) or including both $D_{s}$ and $D$ dressings (dash-dotted line), and, finally, the full calculation that also incorporates the dressing of the baryons (solid line). This is the most interesting of the $C=-1$ sectors considered since the $\bar{D}_{s} N$ system develops in free space a subthreshold bound state at $2906 \mathrm{MeV}$ that couples significatively to $\bar{D}_{s} N$ states. Therefore, this pole will be very sensitive to the medium effects. Indeed, when only Pauli blocking effects are considered, the pole moves about $40 \mathrm{MeV}$ toward higher energy as expected.

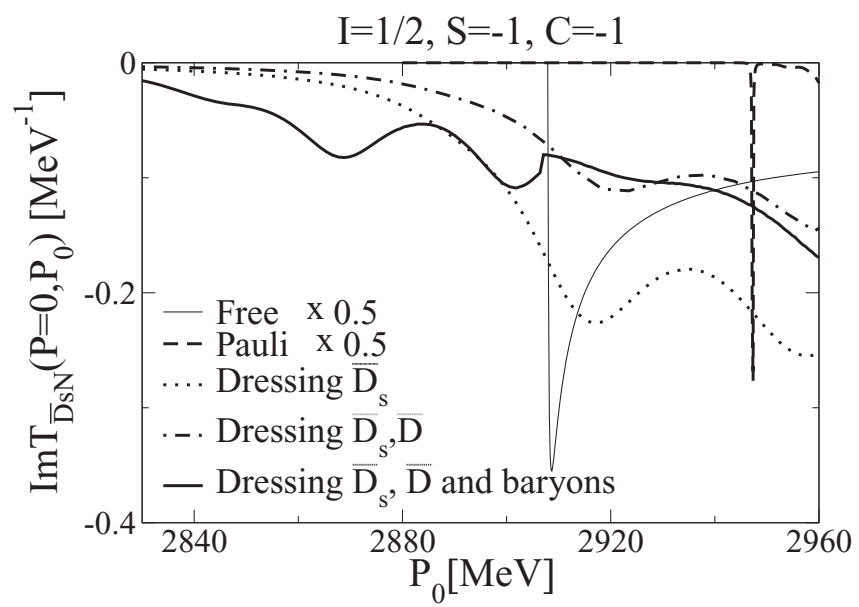

FIG. 3. Imaginary part of the $I=1 / 2, S=-1$, and $C=-1$ $\bar{D}_{s} N \rightarrow \bar{D}_{s} N$ scattering amplitude in nuclear matter at normal saturation density $\rho_{0}$ and zero temperature, as a function of the total energy $P_{0}$, for a total momentum $\vec{P}=0$ and various approximations. 

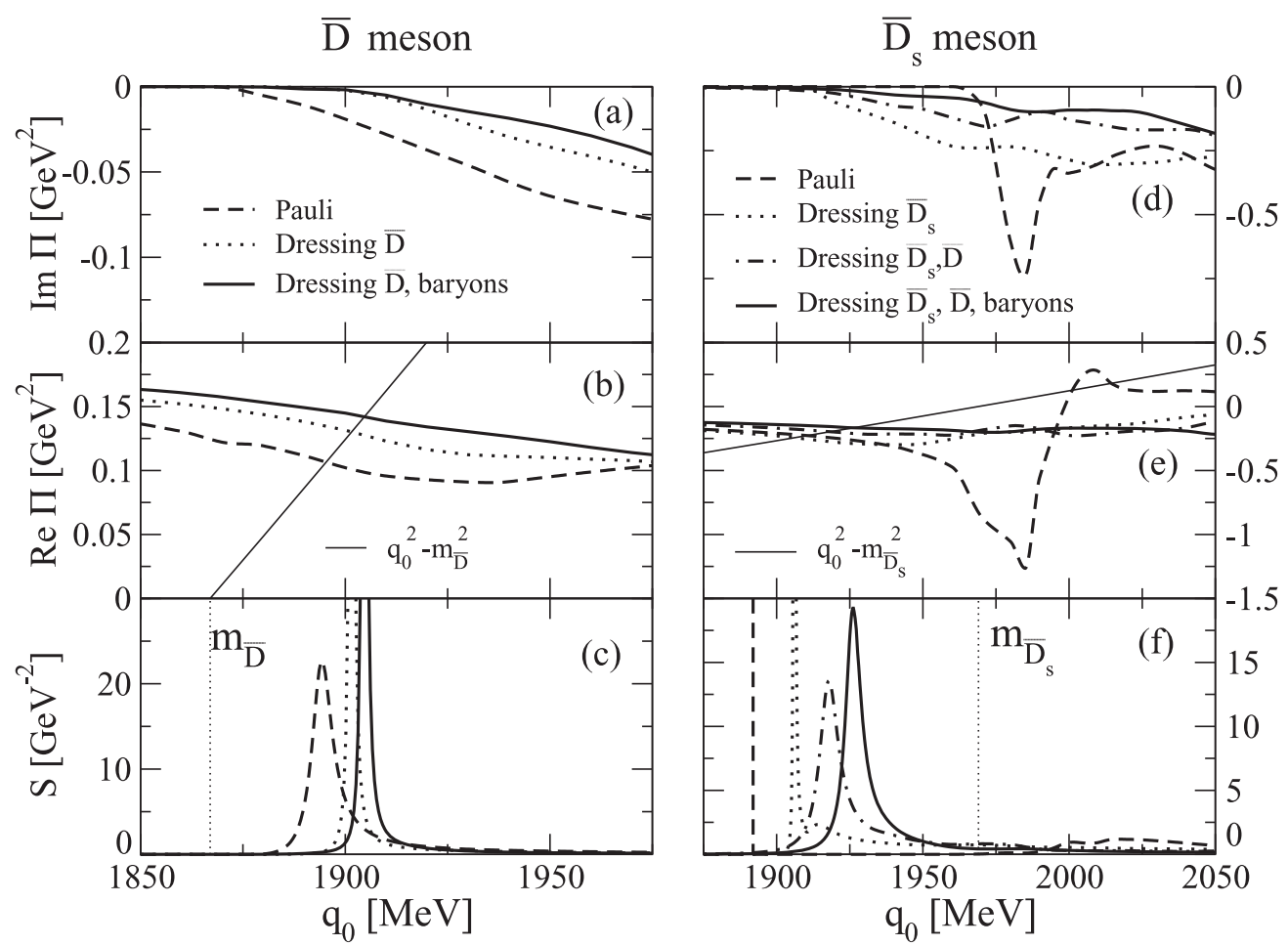

FIG. 4. The same as Fig. 2 for the $\bar{D}$ (left panels) and $\bar{D}_{s}$ (right panels) mesons.

We observe very drastic changes when the dressing of the $\bar{D}$ and $\bar{D}_{s}$ mesons is incorporated. The reason is that, as we will see, the in-medium quasiparticle peak of the $\bar{D}_{s}$ meson experiences a strong attraction. This moves the in-medium threshold for $\bar{D}_{s} N$ states below the position of the resonance, making its decay possible and quite probable due to the significant coupling to these states. When the baryon self-energies are included, the amplitude shifts toward lower energies by an amount consistent with the attraction felt by the dressed baryons.

The $\bar{D}$ and $\bar{D}_{s}$ self-energies and spectral functions are shown in Fig. 4 as functions of $q_{0}$, including Pauli blocking effects, the additional self-consistent dressing of the given meson, and, in the case of the $\bar{D}_{s}$, incorporating also the dressing of the $\bar{D}$ meson in the $\bar{D} Y$ intermediate states coupling to $\bar{D}_{s} N$. Results when baryon dressing is included are also shown. Again the thin solid lines indicate the $q_{0}^{2}-m_{\bar{D}}^{2}$ (left panel) and the $q_{0}^{2}-m_{\bar{D}_{s}}^{2}$ functions (right panel).

The self-energy of the $\bar{D}$ mesons is quite smooth due to the absence of resonances in the $\bar{D} N$ sector. The repulsive character of the $\bar{D} N$ amplitude gives rise to a quasiparticle peak in the $\bar{D}$ spectral function appearing at higher energy than the $\bar{D}$ meson mass, by $27 \mathrm{MeV}$ in the case of considering Pauli blocking effects only, or by $35 \mathrm{MeV}$ when the additional self-consistent dressing of the $\bar{D}$ meson is also taken into account. The repulsive mass shift obtained in Refs. [101,103] is somewhat smaller, of the order of $20 \mathrm{MeV}$ for both cases. On the other hand, the self-energy of the $\bar{D}_{s}$ meson shows a richer structure, which, in the case of Pauli blocking, is seen as a bump in the spectral function around $2000 \mathrm{MeV}$. This is a reflection of the presence, in the $\bar{D}_{s} N$ amplitude, of a pole at $2906 \mathrm{MeV}$, which appears shifted about $40 \mathrm{MeV}$ to higher energies when Pauli blocking effects are incorporated (Fig. 3). The dressing of the $\bar{D}_{s}$ meson smears this structure in such a way that one barely sees any trace of it in the corresponding spectral function. Moreover, the delta-like quasiparticle peak, appearing $77 \mathrm{MeV}$ below the free $\bar{D}_{s}$ mass when only Pauli blocking effects are considered, moves $15 \mathrm{MeV}$ upward in energy when the $\bar{D}_{s}$ meson is dressed. Considering the additional dressing of the $\bar{D}$ meson in the related $\bar{D} Y$ loops produces a substantial change in the $\bar{D}_{s}$ self-energy. This is easy to understand from the results of Table II, where we see that the pole at $2906 \mathrm{MeV}$ couples also very strongly to $\bar{D} Y$ states. The loss of attraction in the region of the quasiparticle peak moves it toward a higher energy. Finally, the effect of including the dressing of the baryons moves the $\bar{D}_{s}$ quasiparticle peak further up, which ends being around $40 \mathrm{MeV}$ below the free mass. Our findings differ again quite strongly from those of Ref. [101], which are dominated by an exotic coupled-channel molecule at $2780 \mathrm{MeV}$ [73], which is the equivalent to the pole at $2906 \mathrm{MeV}$ found in the model of Ref. [105] and used in the present work. As a consequence, the spectral function for the $\bar{D}_{s}$ meson found in Ref. [101] shows two distinct peaks, the quasiparticle one located about $10 \mathrm{MeV}$ above the free $\bar{D}_{s}$ mass, and a narrow resonance-hole mode located $150 \mathrm{MeV}$ below.

In Fig. 5 we display the behavior of the $\Lambda_{c}(2595)$ resonance at two densities, $\rho_{0}$ and $2 \rho_{0}$, and two temperatures, $T=0$ and $100 \mathrm{MeV}$. Results are presented for the complete model that includes the self-consistent meson self-energies and the dressing of the baryons. We observe that the in-medium resonance at $T=0$ appears below the free space position, 


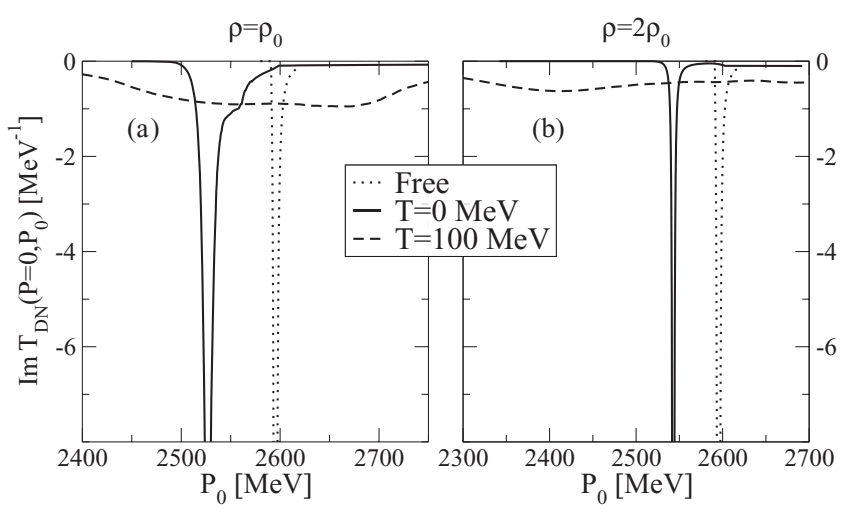

FIG. 5. Imaginary part of the $I=0, D N \rightarrow D N$ scattering amplitudes in nuclear matter at $\rho_{0}$ (left panel) and $2 \rho_{0}$ (right panel), as functions of the total energy $P_{0}$, for a total momentum $\vec{P}=0$ and two temperatures, $T=0$ (solid lines) and $T=100 \mathrm{MeV}$ (dashed lines). The calculation in free space is also given for reference.

by $75 \mathrm{MeV}$ for $\rho_{0}$ and $50 \mathrm{MeV}$ for $2 \rho_{0}$. This behavior can be essentially understood from the stronger Pauli blocking effect at $2 \rho_{0}$. The changes associated with temperature are also very significant. As already seen in Ref. [103], increasing the temperature broadens the $\Lambda_{c}(2595)$ considerably due to the spreading of the $D$-meson strength because of the effect of Fermi motion.

The effect of density and temperature in the spectral functions of the $D, D_{s}, \bar{D}$, and $\bar{D}_{s}$ mesons are shown in Figs. 6 to 9, where we compare results for two temperatures, $T=0$ (solid lines) and $T=100$ (dashed lines), and two densities, $\rho=\rho_{0}$ (upper panels) and $\rho=2 \rho_{0}$ (lower panels), for two values of momentum, $q=0 \mathrm{MeV} / \mathrm{c}$ (left panels) and $q=450 \mathrm{MeV} / \mathrm{c}$ (right panels), in the case of the complete selfconsistent calculation, including Pauli blocking and dressing of mesons and baryons. A common behavior in all spectral functions is that finite temperature moves the quasiparticle

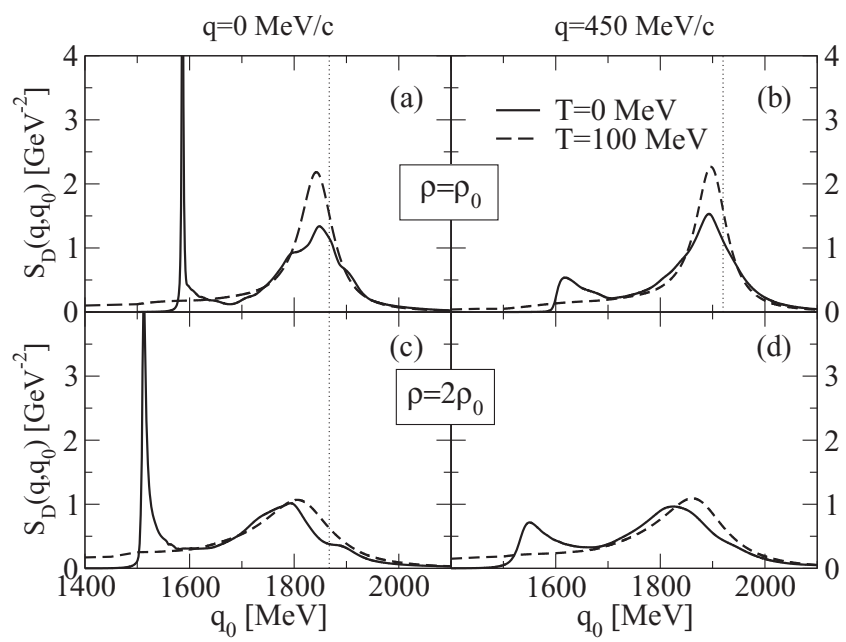

FIG. 6. The spectral function of the $D$ meson at $\rho=\rho_{0}$ (upper panels) and $\rho=2 \rho_{0}$ (lower panels), two temperatures, $T=0$ (solid lines) and $T=100$ (dashed lines), and two values of momentum, $q=0 \mathrm{MeV} / \mathrm{c}$ (left panels) and $q=450 \mathrm{MeV} / \mathrm{c}$ (right panels). Dotted lines indicates the free mass of the meson.

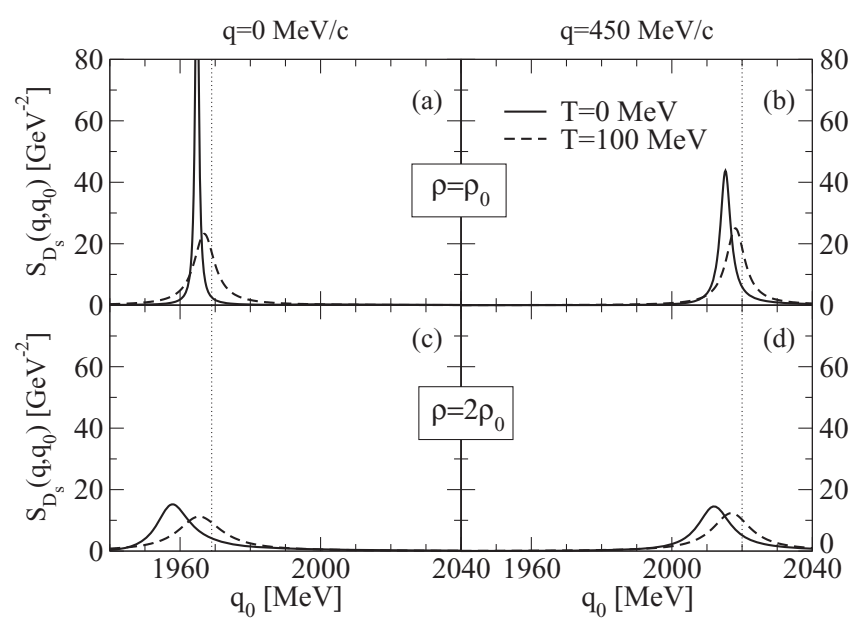

FIG. 7. The same as Fig. 6 for the $D_{s}$ meson.

peak toward its free location. This is a reflection of the reduced size of the self-energy, because, being built up from an average over the smeared thermal Fermi distribution, it involves higher momentum components for which the meson-nucleon interaction is weaker. Except for a few cases, increasing the temperature gives rise to wider quasiparticle peaks because of the increase of collisional width. However, the opposite effect is seen for the $D$ meson in Fig. 6 . As already discussed in Ref. [103], this is due to the fact that the strength under this peak also receives contributions from $\Sigma_{c}(2804) N^{-1}$ hole excitations, which are washed out by temperature as any other resonant-hole mode. Consequently, the peak of the $D$-meson spectral function becomes narrower and more symmetric as temperature increases, similarly to Ref. [103].

The density effects observed in the spectral functions are also clearly understood. In general, we find that the self-energy roughly doubles its size when going from nuclear matter at normal nuclear matter saturation density to a system that is two times denser. This is consistent with the low-density limit behavior and points at a weak density dependence of the in-medium meson-nucleon amplitude in this density region.

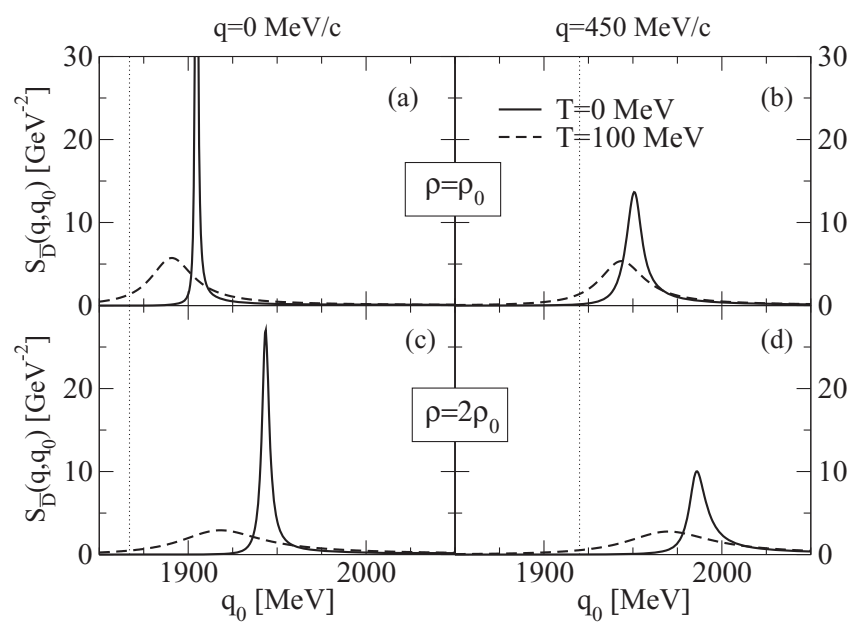

FIG. 8. The same as Fig. 6 for the $\bar{D}$ meson. 


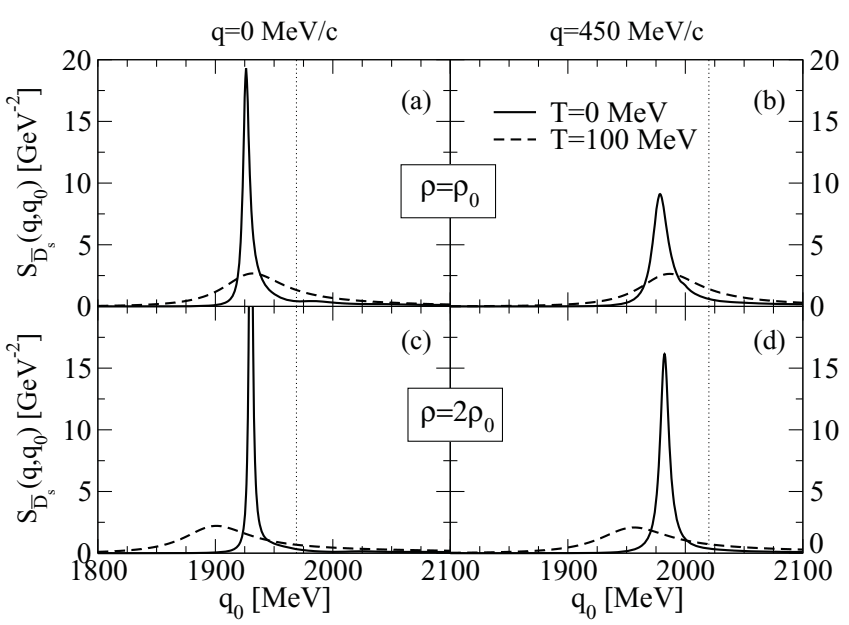

FIG. 9. The same as Fig. 6 for the $\bar{D}_{s}$ meson.

This is the reason why, in general, the quasiparticle peaks of the spectral functions at $2 \rho_{0}$ are found approximately twice farther away from the free space position and are twice wider than in the case of $\rho_{0}$.

\section{SUMMARY AND CONCLUSIONS}

We have studied the properties of open charm mesons, $D, \bar{D}, D_{s}$, and $\bar{D}_{s}$, in nuclear matter at finite temperature within a self-consistent coupled-channel approach that uses, as meson-baryon interaction, a full $t$-dependent vector meson exchange driving force.

The in-medium scattering amplitudes are obtained by solving the Lippmann-Schwinger equation at finite temperature including Pauli blocking effects, the self-consistent $D, \bar{D}, D_{s}$, and $\bar{D}_{s}$ self-energies, paying particular attention to their mutual influence, and the dressing of the baryons.

We have analyzed how our dynamically generated resonances are affected by density and temperature. As in other similar approaches, the resonances that couple strongly to intermediate states involving nucleons move upward in energy when Pauli blocking effects are considered, as a consequence of the loss of phase space. When the self-consistent dressing of the charm mesons is incorporated, the resonances gain attraction again.

We have seen that dressing the $D_{s}$ meson has a nonnegligible effect on the $D N$ amplitude and on the properties of the $D$ meson. Therefore, we conclude that a simultaneous in-medium treatment of both mesons, as the one attempted in the present work, is necessary. Similarly, the in-medium properties of the $\bar{D}_{s}$ and $\bar{D}$ mesons are interrelated and must also be considered together.
The spectral functions of the $D$ and $\bar{D}_{s}$ mesons are quite rich. At $T=0 \mathrm{MeV}$ and normal nuclear matter density one finds a quasiparticle peak located below the corresponding free meson mass, as well as strength associated to resonant-hole excitations, which, in the particular case of the $D$ meson, is clearly visible as a narrow $\Lambda_{c}(2595) N^{-1}$ excitation peak.

In general, increasing the temperature has the effect of moving the quasiparticle peak toward its free location making it wider, as a consequence of a milder meson-baryon interaction and a larger amount of collisions. The exception found for the $D$-meson is naturally explained in terms of the mixing of the quasiparticle peak with a resonant-hole mode.

For the densities explored, up to twice nuclear matter normal saturation density, we have found that the density effects follow the linear behavior expected for the low-density regime: The self-energy roughly doubles its size when going from nuclear matter at normal saturation density to a system that is two times denser, indicating a mild density dependence of the in-medium meson-baryon interaction amplitudes.

The enormous computational effort of the present work, which uses a coupled-channel formalism, an interaction that goes beyond the $t \rightarrow 0$ limit, and the simultaneous consideration of the in-medium $D$ and $D_{s}\left(\bar{D}_{s}\right.$ and $\left.\bar{D}\right)$ meson self-energies, has prevented us from incorporating the coupling to states involving vector-mesons. We are aware that, given the availability of models that permit dealing with these important degrees of freedom, our approach should be extended to the vector mesons such that it also includes, for instance, the $D^{*} N$ and $D_{s}^{*} Y$ channels in the $C=1, S=0$ sector. We hope that, by first identifying which channels play a relevant role and which ones might be omitted, we can make progress toward this goal in the near future.

\section{ACKNOWLEDGMENTS}

This work is partly supported by EU contract No. MRTN-CT-2006-035482 (FLAVIAnet), by contract FIS2008-01661 from MIC (Spain), by Generalitat de Catalunya contract 2009SGR-1289, and by FEDER/FCT (Portugal) under projects PTDC/FIS/113292/2009 and CERN/FP/116366/2010. We acknowledge the support of the European Community-Research Infrastructure Integrating Activity "Study of Strongly Interacting Matter" (HadronPhysics2, Grant Agreement no. 227431) under the Seventh Framework Programme of EU. LT acknowledges support from the Rosalind Franklin Programme of the University of Groningen (the Netherlands) and the Helmholtz International Center for FAIR within the framework of the LOEWE program by the State of Hesse (Germany). This work has been partially completed with the aid of the HPC cluster Millipede of the University of Groningen.
[1] B. Aubert et al. (BABAR Collaboration), Phys. Rev. Lett. 90, 242001 (2003).

[2] R. A. Briere et al. (CLEO Collaboration), Phys. Rev. D 74, 031106 (2006).
[3] P. Krokovny et al. (Belle Collaboration), Phys. Rev. Lett. 91, 262002 (2003).

[4] K. Abe et al. (Belle Collaboration), Phys. Rev. Lett. 92, 012002 (2004). 
[5] S. K. Choi et al. (Belle Collaboration), Phys. Rev. Lett. 91, 262001 (2003).

[6] D. E. Acosta et al. (CDF II Collaboration), Phys. Rev. Lett. 93, 072001 (2004).

[7] V. M. Abazov et al. (D0 Collaboration), Phys. Rev. Lett. 93, 162002 (2004).

[8] B. Aubert et al. (BABAR Collaboration), Phys. Rev. D 71, 071103 (2005).

[9] K. Abe et al. (Belle Collaboration), Phys. Rev. Lett. 98, 082001 (2007).

[10] P. Pakhlov et al. (Belle Collaboration), Phys. Rev. Lett. 100, 202001 (2008).

[11] K. Abe et al. (Belle Collaboration), Phys. Rev. Lett. 94, 182002 (2005).

[12] B. Aubert et al. (BaBar Collaboration), Phys. Rev. Lett. 101, 082001 (2008).

[13] S. Uehara et al. (Belle Collaboration), Phys. Rev. Lett. 96, 082003 (2006).

[14] M. Artuso et al. (CLEO Collaboration), Phys. Rev. Lett. 86, 4479 (2001).

[15] R. Mizuk et al. (Belle Collaboration), Phys. Rev. Lett. 94, 122002 (2005).

[16] C. P. Jessop et al. (CLEO Collaboration), Phys. Rev. Lett. 82, 492 (1999).

[17] S. E. Csorna et al. (CLEO Collaboration), Phys. Rev. Lett. 86, 4243 (2001).

[18] R. Chistov et al. (BELLE Collaboration), Phys. Rev. Lett. 97, 162001 (2006).

[19] B. Aubert et al. (BaBar Collaboration), Phys. Rev. D 77, 012002 (2008).

[20] B. Aubert et al. (BaBar Collaboration), Phys. Rev. Lett. 97, 232001 (2006).

[21] B. Aubert et al. (BaBar Collaboration), Phys. Rev. Lett. 98, 012001 (2007)

[22] R. Mizuk et al. (Belle Collaboration), Phys. Rev. Lett. 98, 262001 (2007).

[23] S. Godfrey and N. Isgur, Phys. Rev. D 32, 189 (1985).

[24] S. Capstick and N. Isgur, Phys. Rev. D 34, 2809 (1986).

[25] J. S. Ball and W. R. Frazer, Phys. Rev. Lett. 7, 204 (1961).

[26] H. W. Wyld, Phys. Rev. 155, 1649 (1967).

[27] R. H. Dalitz, T. C. Wong, and G. Rajasekaran, Phys. Rev. 153, 1617 (1967).

[28] R. K. Logan and H. W. Wyld, Phys. Rev. 158, 1467 (1967).

[29] G. Rajasekaran, Phys. Rev. 5, 610 (1972).

[30] P. B. Siegel and W. Weise, Phys. Rev. C 38, 2221 (1988).

[31] N. Kaiser, P. B. Siegel, and W. Weise, Nucl. Phys. A 594, 325 (1995); Phys. Lett. B 362, 23 (1995); N. Kaiser, T. Waas, and W. Weise, Nucl. Phys. A 612, 297 (1997).

[32] J. Nieves and E. Ruiz Arriola, Phys. Rev. D 63, 076001 (2001).

[33] C. Garcia-Recio, J. Nieves, E. R. Arriola, and M. J. Vicente Vacas, Phys. Rev. D 67, 076009 (2003).

[34] A. Ramos, E. Oset, and C. Bennhold, Phys. Rev. Lett. 89, 252001 (2002).

[35] E. Oset, A. Ramos, and C. Bennhold, Phys. Lett. B 527, 99 (2002).

[36] D. Jido, J. A. Oller, E. Oset, A. Ramos, and U. G. Meissner, Nucl. Phys. A 725, 181 (2003).

[37] E. Oset and A. Ramos, Nucl. Phys. A 635, 99 (1998).

[38] U. G. Meissner and J. A. Oller, Nucl. Phys. A 673, 311 (2000).

[39] J. A. Oller, E. Oset, and A. Ramos, Prog. Part. Nucl. Phys. 45, 157 (2000).
[40] J. A. Oller and U. G. Meissner, Phys. Lett. B 500, 263 (2001).

[41] J. Nieves and E. R. Arriola, Phys. Rev. D 64, 116008 (2001).

[42] M. F. M. Lutz and E. E. Kolomeitsev, Nucl. Phys. A 700, 193 (2002).

[43] M. F. M. Lutz and E. E. Kolomeitsev, Nucl. Phys. A 730, 392 (2004)

[44] T. Inoue, E. Oset, and M. J. Vicente Vacas, Phys. Rev. C 65, 035204 (2002).

[45] J. A. Oller, J. Prades, and M. Verbeni, Phys. Rev. Lett. 95, 172502 (2005).

[46] B. Borasoy, R. Nissler, and W. Weise, Eur. Phys. J. A 25, 79 (2005).

[47] B. Borasoy, U. G. Meissner, and R. Nissler, Phys. Rev. C 74, 055201 (2006).

[48] T. Hyodo, D. Jido, and A. Hosaka, Phys. Rev. C 78, 025203 (2008).

[49] T. Hyodo, D. Jido, and L. Roca, Phys. Rev. D 77, 056010 (2008).

[50] E. E. Kolomeitsev and M. F. M. Lutz, Phys. Lett. B 585, 243 (2004).

[51] S. Sarkar, E. Oset, and M. J. Vicente Vacas, Nucl. Phys. A 750, 294 (2005); 780, 78 (2006).

[52] L. Roca, S. Sarkar, V. K. Magas, and E. Oset, Phys. Rev. C 73, 045208 (2006).

[53] M. Doring, E. Oset, and D. Strottman, Phys. Lett. B 639, 59 (2006).

[54] M. F. M. Lutz, G. Wolf, and B. Friman, Nucl. Phys. A 706, 431 (2002).

[55] C. García-Recio, J. Nieves, and L. L. Salcedo, Phys. Rev. D 74, 034025 (2006).

[56] H. Toki, C. Garcia-Recio, and J. Nieves, Phys. Rev. D 77, 034001 (2008).

[57] P. Gonzalez, E. Oset, and J. Vijande, Phys. Rev. C 79, 025209 (2009).

[58] S. Sarkar et al., Eur. Phys. J. A 44, 431 (2010).

[59] E. Oset and A. Ramos, Eur. Phys. J. A 44, 445 (2010).

[60] A. Martinez Torres, K. P. Khemchandani, and E. Oset, Phys. Rev. C 77, 042203 (2008).

[61] A. Martinez Torres, K. P. Khemchandani, and E. Oset, Eur. Phys. J. A 35, 295 (2008).

[62] K. P. Khemchandani, A. Martinez Torres, and E. Oset, Eur. Phys. J. A 37, 233 (2008).

[63] D. Jido and Y. Kanada-En'yo, Phys. Rev. C 78, 035203 (2008).

[64] Y. Kanada-En'yo and D. Jido, Phys. Rev. C 78, 025212 (2008).

[65] E. E. Kolomeitsev and M. F. M. Lutz, Phys. Lett. B 582, 39 (2004).

[66] J. Hofmann and M. F. M. Lutz, Nucl. Phys. A 733, 142 (2004).

[67] D. Gamermann, E. Oset, D. Strottman, and M. J. Vicente Vacas, Phys. Rev. D 76, 074016 (2007).

[68] D. Gamermann and E. Oset, Eur. Phys. J. A 33, 119 (2007).

[69] F. K. Guo, P. N. Shen, H. C. Chiang, and R. G. Ping, Phys. Lett. B 641, 278 (2006).

[70] F. K. Guo, P. N. Shen, and H. C. Chiang, Phys. Lett. B 647, 133 (2007).

[71] M. F. M. Lutz and E. E. Kolomeitsev, Nucl. Phys. A 730, 110 (2004).

[72] M. F. M. Lutz and E. E. Kolomeitsev, Nucl. Phys. A 755, 29c (2005).

[73] J. Hofmann and M. F. M. Lutz, Nucl. Phys. A 763, 90 (2005).

[74] L. Tolos, J. Schaffner-Bielich, and A. Mishra, Phys. Rev. C 70, 025203 (2004).

[75] T. Mizutani and A. Ramos, Phys. Rev. C 74, 065201 (2006). 
[76] J. Hofmann and M. F. M. Lutz, Nucl. Phys. A 776, 17 (2006).

[77] N. Isgur and M. B. Wise, Phys. Lett. B 232, 113 (1989).

[78] M. Neubert, Phys. Rep. 245, 259 (1994).

[79] A. V. Manohar and M. B. Wise, Heavy Quark Physics, Cambridge Monographs on Particle Physics, Nuclear Physics and Cosmology, Vol. 10 (Cambridge University Press, UK, 2000).

[80] C. Garcia-Recio, V. K. Magas, T. Mizutani, J. Nieves, A. Ramos, L. L. Salcedo, and L. Tolos, Phys. Rev. D 79, 054004 (2009).

[81] D. Gamermann, C. Garcia-Recio, J. Nieves, L. L. Salcedo, and L. Tolos, Phys. Rev. D 81, 094016 (2010).

[82] J.-J. Wu, R. Molina, E. Oset, and B. S. Zou, Phys. Rev. Lett. 105, 232001 (2010).

[83] J. Haidenbauer, G. Krein, U. G. Meissner, and A. Sibirtsev, Eur. Phys. J. A 33, 107 (2007).

[84] J. Haidenbauer, G. Krein, U. G. Meissner, and A. Sibirtsev, Eur. Phys. J. A 37, 55 (2008).

[85] J. Haidenbauer, G. Krein, U. G. Meissner, and L. Tolos, Eur. Phys. J. A 47, 18 (2011).

[86] T. Matsui and H. Satz, Phys. Lett. B 178, 416 (1986).

[87] K. Tsushima, D. H. Lu, A. W. Thomas, K. Saito, and R. H. Landau, Phys. Rev. C 59, 2824 (1999).

[88] C. Garcia-Recio, J. Nieves, and L. Tolos, Phys. Lett. B 690, 369 (2010).

[89] S. J. Brodsky, I. A. Schmidt, and G. F. de Teramond, Phys. Rev. Lett. 64, 1011 (1990).

[90] M. E. Luke, A. V. Manohar, and M. J. Savage, Phys. Lett. B 288, 355 (1992).

[91] K. Tsushima et al., arXiv:1101.3389 [nucl-th].

[92] [http://www.gsi.de/fair/].
[93] K. Tsushima, D. H. Lu, A. W. Thomas, K. Saito, and R. H. Landau, Phys. Rev. C 59, 2824 (1999).

[94] A. Sibirtsev, K. Tsushima, and A. W. Thomas, Eur. Phys. J. A 6, 351 (1999).

[95] A. Hayashigaki, Phys. Lett. B 487, 96 (2000).

[96] A. Mishra, E. L. Bratkovskaya, J. Schaffner-Bielich, S. Schramm, and H. Stöcker, Phys. Rev. C 70, 044904 (2004).

[97] A. Andronic, P. Braun-Munzinger, K. Redlich, and J. Stachel, Phys. Lett. B 659, 149 (2008).

[98] T. Hilger, R. Thomas, and B. Kampfer, Phys. Rev. C 79, 025202 (2009).

[99] A. Mishra and A. Mazumdar, Phys. Rev. C 79, 024908 (2009).

[100] A. Kumar and A. Mishra, Phys. Rev. C 81, 065204 (2010).

[101] M. F. M. Lutz and C. L. Korpa, Phys. Lett. B 633, 43 (2006).

[102] L. Tolos, J. Schaffner-Bielich, and H. Stoecker, Phys. Lett. B 635, 85 (2006).

[103] L. Tolos, A. Ramos, and T. Mizutani, Phys. Rev. C 77, 015207 (2008).

[104] L. Tolos, C. Garcia-Recio, and J. Nieves, Phys. Rev. C 80, 065202 (2009).

[105] C. E. Jiménez-Tejero, A. Ramos, and I. Vidaña, Phys. Rev. C 80, 055206 (2009).

[106] K. Nakamura et al. (Particle Data Group), J. Phys. G 37, 075021 (2010).

[107] R. Machleidt, K. Holinde, and C. Elster, Phys. Rept. 149, 1 (1987).

[108] J. I. Kapusta and C. Gale, Finite Temperature Field Theory and Applications, 2nd ed. (Cambridge University Press, New York, 2006).

[109] T. Matsubara, Prog. Theor. Phys. 14, 351 (1955) 